\title{
Enhancement of Oxygen Reduction Activities by Pt Nanoclusters Decorated on Ordered Mesoporous Porphyrinic Carbons
}

\author{
S.-M. Hwang \\ Submitted to Journal of Materials Chemistry A
}

January 2016

\author{
Chemistry Department
}

Brookhaven National Laboratory

\author{
U.S. Department of Energy \\ [DOE Office of Science]
}

Notice: This manuscript has been authored by employees of Brookhaven Science Associates, LLC under Contract No. DE- SC0012704 with the U.S. Department of Energy. The publisher by accepting the manuscript for publication acknowledges that the United States Government retains a non-exclusive, paid-up, irrevocable, world-wide license to publish or reproduce the published form of this manuscript, or allow others to do so, for United States Government purposes. 


\section{DISCLAIMER}

This report was prepared as an account of work sponsored by an agency of the United States Government. Neither the United States Government nor any agency thereof, nor any of their employees, nor any of their contractors, subcontractors, or their employees, makes any warranty, express or implied, or assumes any legal liability or responsibility for the accuracy, completeness, or any third party's use or the results of such use of any information, apparatus, product, or process disclosed, or represents that its use would not infringe privately owned rights. Reference herein to any specific commercial product, process, or service by trade name, trademark, manufacturer, or otherwise, does not necessarily constitute or imply its endorsement, recommendation, or favoring by the United States Government or any agency thereof or its contractors or subcontractors. The views and opinions of authors expressed herein do not necessarily state or reflect those of the United States Government or any agency thereof. 


\section{Enhancement of Oxygen Reduction Activities by Pt Nanoclusters Decorated on Ordered Mesoporous Porphyrinic Carbons}

Sun-Mi Hwang, ${ }^{\dagger}$ YongMan Choi, ${ }^{\ddagger}$ Min Gyu Kim, ${ }^{\S}$ Young-Jun Sohn, ${ }^{\dagger}$ Jae Yeong Cheon, ${ }^{\prime \prime}$ Sang Hoon Joo, ${ }^{\prime \prime} \perp$ Sung-Dae Yim, ${ }^{\dagger}$ Kurian A. Kuttiyiel, ${ }^{\#}$ Kotaro Sasaki, ${ }^{\#}$ Radoslav R. Adzic, ${ }^{\#}$ and Gu-Gon Park ${ }^{* *}$

${ }^{\dagger}$ Fuel Cell Laboratory, Korea Institute of Energy Research (KIER), Daejeon 305-343, South Korea

${ }^{\ddagger}$ SABIC Technology Center, Riyadh 11551, Saudi Arabia

${ }^{\S}$ Pohang Accelerator Laboratory, Pohang 790-784, Republic of Korea

"Department of Chemistry, Ulsan National Institute of Science and Technology (UNIST), Ulsan 689798, South Korea

${ }^{\perp}$ School of Energy and Chemical Engineering, Ulsan National Institute of Science and Technology (UNIST), Ulsan 689-798, South Korea

"Chemistry Department, Brookhaven National Laboratory, Upton, New York 11973, USA

\section{Abstract}

The high cost of Pt-based membrane electrode assembly (MEA) is a critical hurdle for the commercialization of polymer electrolyte fuel cells (PEFCs). Recently, nonprecious metal-based catalysts (NPMCs) have demonstrated much enhanced activity but their oxygen reduction reaction (ORR) activity is still inferior to that of Pt-based catalyst resulting in much thicker electrode in the MEA. For the reduction of mass transport and ohmic overpotential we adopted a new concept of catalyst that combines ultra-low amount of Pt nanoclusters with metal-nitrogen $\left(\mathrm{M}-\mathrm{N}_{\mathrm{x}}\right)$ doped ordered mesoporous porphyrinic carbon (FeCo-OMPC(L)). The $5 \mathrm{wt} \% \mathrm{Pt} / \mathrm{FeCo}-\mathrm{OMPC}(\mathrm{L})$ showed a 2-fold enhancement in activities compared to a higher loading of Pt. Our experimental results supported by first-principal calculations indicate that trace amount of $\mathrm{Pt}$ nanoclusters on FeCo-OMPC(L) significantly enhances the ORR 
28 activity due to its electronic effect as well as geometric effect from the dual active

29 sites. In terms of fuel cell commercialization, this class of catalyst is a promising 30 candidate due to its limited use of Pt in the MEA.

31 


\section{Introduction}

33 Polymer electrolyte fuel cells (PEFCs) have been recognized as an ideal electrical 34 power system due to its eco-friendly and highly efficient energy conversion 35 characteristics. However, the development of cost effective electrocatalyst by 36 reducing or excluding the platinum in the fuel cell is one of the main challenges for 37 the practical application of PEFCs. ${ }^{1-7}$ After Jasinski’s pioneering work about notable 38 oxygen reduction reaction (ORR) activity on Co-phthalocyanine in alkaline medium, ${ }^{8}$ 39 the class of non-precious metal-based catalysts (NPMCs) has been receiving attention, 40 especially the nitrogen coordinated transition metal (Fe and/or Co) doped carbon

41 compounds $\left(\mathrm{M}-\mathrm{N}_{\mathrm{x}}-\mathrm{C}\right){ }^{9-40}$ The development of NPMCs with remarkable catalytic 42 activity and stability towards the ORR rivaling those of Pt-based catalysts has been a 43 challenging task as it requires fine tuning the composition of transition metal and 44 nitrogen coordination number. The ORR activity of $\mathrm{M}-\mathrm{N}_{\mathrm{x}}-\mathrm{C}$ catalysts strongly 45 depends on the types of nitrogen and transition metal sources, heat treatment 46 temperature, and graphiticity and morphology of carbon support, etc. ${ }^{17-40}$ Even though 47 the ORR activity and durability in alkaline media have been improved via the 48 optimization of the synthetic process, ${ }^{33-34}$ the ORR activity in acid medium is still 49 inferior to Pt-based electrocatalysts. Recently, Dodelet et al. ${ }^{41}$ and Joo et al. ${ }^{40}$ 50 reported high ORR activity for NPMC, i.e. Fe- and/or Co-N-C catalyst and self51 supported ordered mesoporous porphyrinic carbon (OMPC), which shows a 52 commensurate ORR activity with $\mathrm{Pt} / \mathrm{C}$ in acidic electrolyte using rotating disk 53 electrode (RDE).

54 In terms of membrane electrode assembly (MEA) fabrication, however, NPMC55 based MEA has around ten times thicker catalyst layer than that of conventional Pt/C 56 MEA $^{41}$ due to high catalyst loading resulting in increased mass transport resistance as 
57 well as electrical resistance. The high ORR activity observed at a half-cell

58 configuration could not be observed in NPMC-based MEA. Hence, decreasing the

59 catalyst loading in the unit area may be important to obtain consistent performance in

60 MEA, as shown in the RDE evaluation.

61 In this study, we evaluate a new concept that can enhance the ORR activity via using

62 a low-amount of platinum nanocluster on NPMC (Pt/NPMC hybrid catalyst). The

63 Pt/NPMC hybrid catalyst shows much enhanced ORR activity due to its electronic

64 effect and also due to the geometric effect caused from dual active sites. The density-

65 functional-theory (DFT) calculations suggest the mechanism of Pt cluster formation

66 on the NPMC and also explain its positive effect on ORR that is constrained by its 67 loading.

68 


\section{Experimental Section}

\subsection{Material synthesis}

Nitrogen coordinated transition metal based ordered mesoporous carbon was synthesized by a nanocasting method. ${ }^{40 ; 42-44}$ Ordered mesoporous silica (OMS(L)) templates with ca. $20 \mathrm{~nm}$ large mesopores were well mixed with 5,10,15,20tetrakis(4-methoxyphenyl)-21H,23H-porphine iron(III) chloride (Fe-TMPPCl) and 5,10,15,20-tetrakis(4-methoxyphenyl)-21H,23H-porphine cobalt(II) (Co-TMPP) as a metalloporphyrinic precursors, then the mixture was pyrolyzed at $800{ }^{\circ} \mathrm{C}$ for $3 \mathrm{~h}$ in $\mathrm{N}_{2}$ atmosphere. The silica templates and metal particles in the composite were removed by washing in $10 \%$ HF solution, then the resulting FeCo-OMPC(L) was washed with deionized water for several times, followed by drying at $80{ }^{\circ} \mathrm{C}$ overnight. The OMPC(L) without transition metals was also prepared with 5,10,15,20-tetrakis(4methoxyphenyl)-21H,23H porphyrin (TMPPH) and OMS(L) template under the same pyrolysis condition as described before. ${ }^{40} \mathrm{Pt} / \mathrm{FeCo}-\mathrm{OMPC}(\mathrm{L})$ electrocatalysts with various Pt loading were prepared by sodium borohydride $\left(\mathrm{NaBH}_{4}\right)$ reduction method. ${ }^{45-47}$ FeCo-OMPC(L) was well dispersed in DI-based $\mathrm{H}_{2} \mathrm{PtCl}_{6}$ solution, then excess of $0.5 \mathrm{M} \mathrm{NaBH}_{4}$ solution was added drop by drop into the mixture with vigorous stirring for the complete reduction of the $\mathrm{PtCl}_{6}{ }^{2-}$ ion to $\mathrm{Pt}$ metal. The mixture was stirred for $24 \mathrm{~h}$ at ambient temperature, and washed with excess deionized water and dried at $80{ }^{\circ} \mathrm{C}$ for $24 \mathrm{~h}$. For the direct comparison of ORR activities, catalysts that have the same Pt loading were prepared by using FeCo-OMPC(L), OMPC(L) and Vulcan XC-72 as supports using the same procedures.

\subsection{Characterization}

The morphology and distribution of Pt nanoclusters in the Pt/FeCo-OMPC(L), Pt/OMPC(L), and Pt/C catalysts were examined by field emission transmission 
94 electron microscope (FE-TEM, Tecnai TF30 ST, FEI). High-angle annular dark field

95 (HAADF) images and EDS-mapping of FeCo-OMPC(L) and Pt/FeCo-OMPC(L) was

96 observed by Titan Double Cs corrected TEM (Titan cubed G2 60-300, FEI). X-ray

97 diffraction (XRD, D/MAX-2500, RIGAKU) patterns of the prepared catalysts were

98 obtained to calculate the Pt particle size using Scherrer's equation. The surface area

99 and the pore size distribution of the catalysts were measured by $\mathrm{N}_{2}$ adsorption-

100 desorption isotherms (ASAP-2020, Micrometrics). The metal contents of Pt, Fe, and

101 Co were measured by inductively coupled plasma atomic emission spectroscopy

102 (ICP-AES), and the composition of $\mathrm{C}$ and $\mathrm{N}$ elements was examined with elemental

103 analyzer (EA). X-ray photoelectron spectroscopy (XPS) was performed on AXIS

104 NOVA with monochromated $\mathrm{Cu} \mathrm{K}_{\alpha} \mathrm{X}$-ray source.

105 In situ electrochemical cell for X-ray absorption spectroscopy (XAS) analysis was 106 specially designed to operate at potentiostatic mode. Teflon based in situ XAS cell 107 was mounted on a goniometer stage passivated implanted planar silicon (PIPS, 108 Canberra Co.) and the fluorescence detector was positioned on top of the 109 electrochemical cell (Fig. S7). XAS signals of Fe k-edge, Co k-edge, and Pt $\mathrm{L}_{3}$-edge

110 for the catalysts were recorded in fluorescence mode on the BL10C beam line at the 111 Pohang Light Sources (PLS) with a ring current of $200 \mathrm{~mA}$ at $3.0 \mathrm{GeV}$. The 112 monochromatic X-ray beam could be obtained using a liquid nitrogen-cooled Si (111) 113 double crystal monochromator. Working electrode for the in situ XAS analysis was 114 prepared by spray coating the Nafion containing catalyst ink onto the Nafion 117 115 membrane. Precise energy calibration was simultaneously carried out for each 116 measurement with each reference metal foil placed in front of the third ion chamber 117 using partially-bypassed X-ray beam. The data processing of the experimental spectra 
to normalized XANES and Fourier-transform (FT) EXAFS were performed through

119 the standard XAFS procedure. ${ }^{48-51}$

\section{$120 \quad$ 2.3. Electrochemical analysis}

121 The ORR kinetics on Pt/FeCo-OMPC(L) catalysts were examined using rotating 122 disk electrode (RDE) method in a typical three-electrodes cell with a Pt counter 123 electrode and hydrogen reference electrode (Gaskatel GmbH, Germany). The catalyst 124 ink was prepared by sonication of $30 \mathrm{mg}$ of Pt/FeCo-OMPC(L) in $1.2 \mathrm{ml}$ of ethanol 125 and DI mixture with $0.02 \mathrm{ml}$ of $5 \mathrm{wt} \%$ Nafion solution. A catalyst ink was pipetted 126 onto a glassy carbon (GC, $0.196 \mathrm{~cm}^{2}$ ) disk electrode mounted on the inverted rotator 127 under rotating at $300 \mathrm{rpm}$ to make uniform thin film, then dried under rotation for 20 $128 \mathrm{~min}$ at room temperature. ${ }^{52}$ The total amount of catalyst loading was $84 \mu \mathrm{g}_{\text {catalyst }} / \mathrm{cm}^{2}$, 129 but the Pt mass loading was dependent on the Pt contents in the Pt/FeCo-OMPC(L). 130 The catalyst film coated on the GC electrode was cleaned and activated in nitrogen 131 saturated $0.1 \mathrm{M} \mathrm{HClO}_{4}$ electrolyte by cyclic voltammetry between $0 \mathrm{~V}$ and $1.2 \mathrm{~V}$ vs. 132 RHE with a scan rate of $100 \mathrm{mV} / \mathrm{s}$ for 50 cycles until obtaining quasi steady-state I-V 133 characteristic. The ORR kinetics was examined by linear sweep voltammetry (LSV) 134 using anodic sweep from $0.05 \mathrm{~V}$ to $1.15 \mathrm{~V}$ vs. RHE with a sweep rate of $10 \mathrm{mV} / \mathrm{s}$ in 135 oxygen saturated $0.1 \mathrm{M} \mathrm{HClO}_{4}$ as a function of RDE rotation rates from $400 \mathrm{rpm}$ to $1362,500 \mathrm{rpm}$.

\section{2.4. DFT calculation}

138 Periodic plane-wave DFT calculations were performed using the Vienna ab initio 139 simulation package (VASP) $)^{53-54}$ with projector augmented wave (PAW) potentials ${ }^{55}$ 140 and the Perdew-Burke-Ernzerhof (PBE) exchange-correlation functional. ${ }^{56}$ The spin141 polarization method was used in the calculations to correctly account for magnetic 142 properties. Monkhorst-Pack ${ }^{57}$ mesh k-points of $(3 \times 3 \times 3)$ and $(3 \times 3 \times 1)$ for bulk 
143 and surface calculations, respectively, were used. The plane-wave cutoff energy was 144 optimized at $415 \mathrm{eV}$. To describe the interaction between Pt cluster and surface, 145 adsorption energy is defined as $\mathrm{E}_{\mathrm{ads}}=\mathrm{E}\left[\mathrm{Pt}_{\mathrm{n}} /\right.$ surface $]-\mathrm{E}\left[\mathrm{Pt}_{\mathrm{n}}\right]-\mathrm{E}$ [surface $]$, where $146 \mathrm{E}\left[\mathrm{Pt}_{\mathrm{n}} /\right.$ surface $], \mathrm{E}\left[\mathrm{Pt}_{\mathrm{n}}\right]$, and $\mathrm{E}[$ surface $]$ are the calculated electronic energies of 147 adsorbed $\mathrm{Pt}_{\mathrm{n}}$ on a surface, pure $\mathrm{Pt}_{\mathrm{n}}$ cluster, and a clean surface, respectively. Also, we 148 calculated the binding energy of atomic oxygen (BO) as a descriptor for scaling the 149 ORR activity. ${ }^{58-60} \mathrm{BO}$ is defined as $\mathrm{BO}=\mathrm{E}[\mathrm{O} /$ surface $]-\mathrm{E}[$ surface $]-\mathrm{E}[\mathrm{O}]$, where $150 \mathrm{E}[\mathrm{O} /$ surface $]$ and $\mathrm{E}[\mathrm{O}]$, respectively, are the calculated electronic energies of 151 adsorbed oxygen atom on a surface and a triplet oxygen atom. Since the topmost 152 layer's Co metal center of the surface model of Pt/FeCo-OMPC(L) (Fig. S3) was 153 replaced by Pt to support the experimental observation using the EXAFS analysis, we 154 compared BO only on the Fe metal center. The vacancy formation energy of the metal 155 center $^{61}$ is defined as $\mathrm{E}_{\mathrm{M} \text {-vac }}=\mathrm{E}[\mathrm{d}$-surface $]+\mathrm{E}[\mathrm{M}]-\mathrm{E}[\mathrm{p}$-surface $]$, where d-surface 156 and p-surface are the surfaces with and without an $\mathrm{M}$ vacancy ( $\mathrm{M}=\mathrm{Co}$ or $\mathrm{Fe}$ ). In this 157 study, we used gas-phase atomic metals' energies by considering the number of their 158 unpaired electrons. Incorporation energy of Pt into the vacancy of the metal centers is 159 calculated by $\mathrm{E}_{\mathrm{Pt} \text {-incorp }}=\mathrm{E}[$ Pt-surface $]-\mathrm{E}[\mathrm{Pt}]-\mathrm{E}[\mathrm{d}$-surface $]$, where Pt-surface is the 160 surface after incorporation of Pt into the M vacancy. Similar to the previous study, ${ }^{40}$ 161 the surface calculations on the perfect FeCo-OMPC(L) surface were first carried out 162 on a C, N, and M-terminated (0001) plane (120 C, 12 N, 14 O, 2 Fe, 2 Co, and 2 H 163 atoms) based on its bulk structure (Fig. S4) with a vacuum space of $10 \AA$. Only the 164 bottom bilayer of the 2-layer FeCo-OMPC(L) surface was fixed, and the top bi-layer 165 and the adsorbates were fully relaxed. Then, the M metal center on the topmost layer 166 was removed and replaced by Pt to examine the vacancy formation and incorporation 167 energies. 


\section{Results and Discussion}

Figure 1(a) shows schematic of the synthetic procedure of Pt/FeCo-OMPC(L) via nanocasting method using large pore OMS(L) hard template (Fig. 1(b)) and $\mathrm{NaBH}_{4}$ reduction. EDS mapping images for FeCo-OMPC(L) (Fig. 1(e)) show uniformly distributed Fe, Co, and $\mathrm{N}$ elements in the mesoporous carbon structures with a 7-8 nm pore size (Fig. 1(c)). The FeCo metallic nanoparticles were removed by the HF washing procedure. The final FeCo-OMPC(L) consisted of $1.63 \mathrm{wt} \% \mathrm{Fe}, 1.47 \mathrm{wt} \%$ Co, and $6.8 \mathrm{wt} \% \mathrm{~N}$, as summarized in Table 1. Pt nanoclusters were well distributed on FeCo-OMPC(L) as shown in Fig. 1(d). EDS mapping images for Pt/FeCoOMPC(L) catalyst as shown in Fig. 1(f) clearly demonstrates the presence of Fe, Co and $\mathrm{N}$ elements along with some Pt clusters. The average Pt particle sizes calculated with Pt (111) diffraction peaks from XRD were $2.1 \mathrm{~nm}$ for Pt/FeCo-OMPC(L), 3.5 $\mathrm{nm}$ for Pt/OMPC(L), and $6.8 \mathrm{~nm}$ for Pt/C, respectively (Table S1). Pt lattice fringe was observed with (111) spacing of $2.27 \AA\left(\mathrm{Pt}_{111}\right.$, bulk $\left.=2.26 \AA\right)$ that is in good agreement with the $2.26 \AA$ calculated from Pt(111) diffraction peaks. The metal contents in the Pt/FeCo-OMPC(L) catalysts were summarized in Table 1 . The Fe, Co, and $\mathrm{N}$ elemental composition shows a slight change in weight content before and after Pt loading by $\mathrm{NaBH}_{4}$ reduction.

Figure 2(a) shows the ORR polarization curves for the as-prepared catalysts measured in $0.1 \mathrm{M} \mathrm{HClO}_{4}$ using the $\mathrm{RDE}$ technique. The half-wave potential on Pt/FeCo-OMPC(L) was $858 \mathrm{mV}$ vs. RHE which was $13 \mathrm{mV}$ and $103 \mathrm{mV}$ higher than that of Pt/OMPC(L) and Pt/C catalysts, respectively. The Pt/FeCo-OMPC(L) showed 1.4 and 8.3-fold enhanced kinetic activity compared to Pt/OMPC(L) and Pt/C at $0.9 \mathrm{~V}$ vs. RHE, respectively (Table S1). The specific activity and mass activity for Pt/FeCoOMPC(L) were also improved by a factor of 2 and 7 relative to those of Pt/OMPC(L) 
and $\mathrm{Pt} / \mathrm{C}$ at $0.9 \mathrm{~V}$, respectively (Fig. 2(b)). This change in ORR activity is clearly due to interaction between Pt nanoclusters and the support. The ORR activity of the support catalysts followed the same order; FeCo-OMPC(L) > OMPC(L) > Vulcan XC-72 (VC) (Fig. S1).

198 A substantial increase in the ORR activity was observed with the introduction of Pt 199 nanoclusters on the FeCo-OMPC(L) (Fig. 3(a)). The positive shift of half-wave 200 potential $\left(E_{1 / 2}\right)$ was an evidence for the enhancement of ORR activity with the 201 different Pt loading on the FeCo-OMPC(L) support. The mass transport resistance 202 corrected ORR kinetics increased by a factor of 9 for $5 \mathrm{wt} \% \mathrm{Pt} / \mathrm{FeCo}-\mathrm{OMPC}(\mathrm{L})$ 203 compared to that of FeCo-OMPC(L) at $0.9 \mathrm{~V}$ vs. RHE. When the Pt-based mass 204 activity was compared with commercial $40 \mathrm{wt} \% \mathrm{Pt} / \mathrm{C}$ catalyst, $5 \mathrm{wt} \% \mathrm{Pt} / \mathrm{FeCo}-$ 205 OMPC(L) showed ca. 2-fold enhanced mass activity at $0.9 \mathrm{~V}$ as shown in Fig. 3(b). 206 Electrochemical surface area (ECSA) of Pt measured by CO stripping increased with 207 Pt metal loading, however, the overall ORR activity was not linearly improved in 208 accordance with the amount of the Pt nanoclusters on the FeCo-OMPC(L).

209 The above results suggest that the enhancement of the ORR activity on Pt/FeCo210 OMPC(L) can be attributed to not only dual active sites but also the electronic effect 211 between Pt nanoparticles and FeCo-OMPC(L). Prior to figuring out the electronic 212 effect, we carefully considered the dual active site effect originating due to the 213 loading of Pt on FeCo-OMPC(L) support. The high surface area of FeCo-OMPC(L) is 214 one of the main factors for the high activity towards the ORR. However, we did 215 observe that a surface area of the Pt/FeCo-OMPC(L) catalysts decreased from 970 to $216458 \mathrm{~m}^{2} / \mathrm{g}$ with an increase in Pt loading, as plotted in Fig. 4. Decrease in the surface 217 area of Pt/FeCo-OMPC(L) can be explained by blocking of the mesopores and 218 micropores in the FeCo-OMPC(L) by 2-3 nm Pt nanoclusters as well as by the 
219 different density of materials. Total pore volume of the catalysts was also decreased 220 from 1.78 to $1.06 \mathrm{~cm}^{3} / g$ with an increase in the Pt loading amount. In other words, the 221 activity loss of the $10 \mathrm{wt} \% \mathrm{Pt} / \mathrm{FeCo}-\mathrm{OMPC}(\mathrm{L})$ may be caused by the decrease of the 222 absolute number of active site. Therefore, the activity enhancement due to the 223 increase in Pt loading was limited by the loss of FeCo-OMPC(L) active area caused 224 by the increase in the number of Pt nanoclusters.

225 In order to understand the electronic effect between the Pt nanoclusters and 226 transition metals (Fe and/or Co) or $\mathrm{N}$ in FeCo-OMPC(L) we carried out XPS, in situ 227 EXAFS analyses and DFT calculations. Figure 5 shows the XPS result for the FeCo228 OMCP(L) and Pt/FeCo-OMPC(L) catalysts. The N 1s XPS spectrum for all catalysts 229 indicate three dominant nitrogen species at 398.6, 400.1, and $401.5 \mathrm{eV}$ associated 230 with pyridinic N (N1), pyrrolic N (N2), and graphitic N (N3), respectively. ${ }^{25}$ Binding 231 energy (BE) shift to lower energy was observed for the graphitic N (N3) for 5 wt\% $232 \mathrm{Pt} / \mathrm{FeCo}-\mathrm{OMCP}(\mathrm{L})$ which is associated with modification of electronic structure of $\mathrm{N}$ 233 site binding with Fe or Co. In contrast, no binding energy shift was examined for 234 pyridinic $\mathrm{N}(\mathrm{N} 1)$ and pyrrolic $\mathrm{N}$ (N2) peaks for the catalysts. As the Pt loading 235 increased, lower shift of BE for Pt $4 \mathrm{f}_{7 / 2}$ was observed indicating an increase in Pt-Pt 236 interaction. The XPS results showed that the electronic structures of Pt nanoclusters 237 on FeCo-OMPC(L) were modified by $\mathrm{N}$ elements connected with transition metals in 238 the FeCo-OMPC(L).

239 We investigated the effect of Pt nanoclusters on the electronic structure of the 240 FeCo-OMPC(L) with in situ XAFS analysis. In situ XAFS analysis was performed at 241 constant potential mode $(0.3 \mathrm{~V}$ vs. RHE) to eliminate the oxygen molecule 242 coordinated with the central Fe and/or Co in the axial direction and the surface of Pt 243 in the Pt/FeCo-OMPC(L). The XANES spectra revealed that the square planar $\mathrm{D}_{4 \mathrm{~h}}$ 
244 structure of Co-TMPP and Fe-TMPPCl compounds was broken down or distorted in 245 the FeCo-OMPC(L) catalyst during the pyrolysis process at high temperatures (Fig.

246 S2). The FT EXAFS for Fe k-edge, Co k-edge of FeCo-OMPC(L) indicated no 247 existence of Fe or Co metallic phase in the FeCo-OMPC(L) structure which is in good 248 agreement with the TEM-EDS mapping images (Fig. 6(b) and 6(c)). There is no 249 evident FT peaks related with Pt-TM coordination (TM=Fe, Co), whereas Pt-N 250 coordination was observed at around 1.93 - $2.01 \AA$ from FT EXAFS for Pt $\mathrm{L}_{3}$-edge, as 251 shown in Fig. 6(a). The peak intensity for Pt-N coordination decreased with an 252 increase in Pt amount in the Pt/FeCo-OMPC(L). This means that the Pt nanoclusters 253 are mainly positioned at near $\mathrm{N}$-site and the interaction between Pt nanoclusters and $\mathrm{N}$ 254 becomes weaker with an increase in the Pt loading in the Pt/FeCo-OMPC(L) 255 catalysts. A slight elongation of Fe-N distance was observed, whereas the Co-N 256 distance had no big change with Pt contents.

257 To support our experimental observations, we carried out DFT calculations using 258 the FeCo-OMPC(L) bulk structure consisting of 152 atoms $(120 \mathrm{C}, 12 \mathrm{~N}, 14 \mathrm{O}, 2 \mathrm{Fe}$, 2592 Co, and $2 \mathrm{H}$ ). As discussed earlier, the EXAFS analysis clearly revealed no 260 interaction between $\mathrm{Pt}$ and the metal centers on Pt/FeCo-OMPC(L) catalyst. To 261 propose a plausible model and mechanism for the Pt nanocluster formation on FeCo262 OMPC(L), we prepared the FeCo-OMPC(L) bulk structure, similar to the previous 263 study (Fig. S4). ${ }^{40}$ The model using the PBE exchange-correlation functional is in good 264 agreement with the EXAFS and HRTEM results, such as the interlayer distance 265 (Expt.: $4.77 \AA$ and DFT: $\sim 4.5 \AA$ ). ${ }^{40}$ To match with the averaged coordination number 266 of $\mathrm{M}-\mathrm{N}(\mathrm{N}=3.3)$ for FeCo-OMPC(L) in Table 2, we kept $\mathrm{N}=3$ by removing one 267 coordinated $\mathrm{N}$ with each metal center. Also, the M-N distances of the bulk model are 268 in agreement with experiment (Co-N: $1.84 \AA$ versus $1.76 \AA$ and $\mathrm{Fe}-\mathrm{N}: 1.83 \AA$ versus 
$2691.74 \AA$ ) (Table 2 and S2). Using the bulk structure, the C, N, Fe, and Co-terminated 270 (0001) surface was generated for the surface calculations (Fig. S3). Then, we

271 calculated the vacancy formation energies for the metal centers. As summarized in 272 Table S2, the Co site can be more easily removed than the Fe site (6.09 eV/atom 273 versus $6.74 \mathrm{eV} /$ atom, respectively). Especially, the strong incorporation strength of Pt 274 into the vacancy indicates that the first step of the Pt nanocluster formation on FeCo275 OMPC(L) may be the incorporation process on the surface vacancy after leaching out 276 of unstable surface metal centers. The essential energy can be provided by the 277 exothermic reduction reaction using $\mathrm{NaBH}_{4}$ as discussed in the experimental section. 278 We observed that the Fe-N distance is lengthened by $2.2 \%$ after the addition of Pt 279 (DFT: $1.84 \AA$ versus $1.87 \AA$ and Expt.: $1.74 \AA$ versus 1.92 - $1.99 \AA$ (Table 2 and S3). 280 Also, the calculated distances of Pt-N and Pt-Pt for $\mathrm{Pt}_{19} / \mathrm{FeCo}-\mathrm{OMPC}(\mathrm{L})$ support 281 experiment (DFT: $1.97 \AA$ versus $2.64 \AA$, respectively, and Expt.: $1.93-2.01 \AA$ versus $2822.85-2.86 \AA$ ) (Table 2 and S3). According to the reasonable agreement with the 283 modeling and experimental results, we propose a mechanism of the growth of $\mathrm{Pt}$ 284 nanoclusters on FeCo-OMPC(L). As shown in Fig. 7 and S5, the first step is the 285 removal of unstable surface metal centers, preferentially Co, and the incorporation of 286 Pt into the metal-center vacancy. Then, the subsequent Pt nanocluster growth may 287 occur via the linkage of the replaced Pt center (Fig. S5). It is desirable to execute 288 more size-dependent studies, but due to high computational time, we optimized only 289 two cases of $\mathrm{Pt}_{6}$ and $\mathrm{Pt}_{19}$ (Fig. S6). It was found that the adsorption energy of $\mathrm{Pt}$ 290 nanoclusters becomes weakened as the nanoclusters grow $\left(\mathrm{Pt}_{6}:-5.21 \mathrm{eV}\right.$ and $\mathrm{Pt}_{19}$ : $2914.31 \mathrm{eV}$ ). Using the perfect FeCo-OMPC(L) model, we found that the longitudinal M292 M distances range from $~ 0.8$ to $1.5 \mathrm{~nm}$. Thus, if we assume that the Pt nanoclusters of $293 \sim 2 \mathrm{~nm}$ in diameter is sphere-like, the possibility to link Pt and the metal center is low 
294 within the calculated M-M distance range, supporting the EXAFS's analysis. In 295 addition to the mechanistic study, we calculated BO by placing one oxygen atom only 296 at the Fe metal center on the surface because of the surface (Pt/FeCo-OMPC(L) def) 297 doesn't have the Co metal center. The Pt/FeCo-OMPC(L) def catalyst $(-2.86 \mathrm{eV})$ 298 showed a slight decrease in BO from that of FeCo-OMPC $(\mathrm{L})_{\mathrm{def}}(-3.10 \mathrm{eV})$, indicating 299 an enhanced ORR activity by the Pt nanocluster on FeCo-OMPC(L) (Fig. 7(d)).

300 From the results, we verified that the enhancement of the ORR mass activity on $301 \mathrm{Pt} / \mathrm{FeCo}-\mathrm{OMPC}(\mathrm{L})$ was attributed to both the electronic effect and the geometric 302 effect caused by dual active sites of Pt nanoclusters and FeCo-OMPC(L). Therefore, 303 the best electrochemical activity towards ORR was exhibited on $5 \mathrm{wt} \% \mathrm{Pt} / \mathrm{FeCo}-$ 304 OMPC(L) considering the complementary effect between the absolute number of the 305 active sites and electronic effect.

\section{Conclusions}

We reported a new approach for the enhanced ORR activity as well as the reduction of the precious metal utilization for fuel cell commercialization. We have synthesized a new class of electrocatalysts consisting of a trace amount of $\mathrm{Pt}$ nanoclusters on $\mathrm{M}-\mathrm{N}$ doped ordered mesoporous porphyrinic carbon to enhance the

312 intrinsic catalytic activity. The FeCo-OMPC(L) itself exhibits ORR activity due to its 313 nitrogen coordinated metal centers, however, by adding low amount of $\mathrm{Pt}$ 314 nanoclusters on FeCo-OMPC(L), much improved ORR activity could be obtained. 315 Both the electronic and geometric effects were observed as the reason of activity 316 enhancement. The $5 \mathrm{wt} \% \mathrm{Pt} / \mathrm{FeCo}-\mathrm{OMPC}(\mathrm{L})$ electrocatalyst indicated a factor of two 317 improvement in the ORR mass activity compared to Pt/C. The FeCo-OMPC(L) Pt 318 loaded catalysts greater than $5 \mathrm{wt} \%$ showed decreased mass activities because of the 
319 reduced electronic effect and the shrunken absolute number of active sites caused by 320 increased Pt particle size and density.

321 


\section{Associated content}

323 Supporting information

324

325 Author information

326 Corresponding Author: gugon@kier.re.kr

327

328 Acknowledgement

329 This research was supported by the New \& Renewable Energy Core Technology

330 Program of the Korea Institute of Energy Technology Evaluation and Planning

331 (KETEP) granted financial resource from the Ministry of Trade, Industry \& Energy, 332 Republic of Korea (No. 20133030011320) and Research and Development Program 333 of the Korea Institute of Energy Research (KIER) (B5-2422-02). This work was also 334 performed in collaboration with Brookhaven National laboratory under contract no. 335 DE-SC0012704. DFT calculations were performed at KAUST Supercomputing 336 Laboratory and the National Energy Research Scientific Computing Center (Contract 337 No. DE-AC02-05CH11231). 
$340 \quad$ 1. Debe, M. K., Nature 2012, 486, 43-51.

341 2. Gasteiger, H. A.; Kocha, S. S.; Sompalli, B.; Wagner, F. T., Appl. Catal. B: Environ. 2005, 56, 9-35.

3. Gasteiger, H. A.; Markovic, N. M., Science 2009, 324, 48-49.

4. Morozan, A.; Jousselme, B.; Palacin, S., Energy Environ. Sci. 2011, 4, 12381254.

5. Brouzgou, A.; Song, S. Q.; Tsiakaras, P., Appl. Catal. B: Environ. 2012, 127, 371-388.

6. Nie, Y.; Li, L.; Wei, Z., Chem. Soc. Rev. 2015, 44, 2168-2201.

7. Stamenkovic, V. R.; Mun, B. S.; Arenz, M.; Mayrhofer, K. J. J.; Lucas, C. A.; Wang, G.; Ross, P. N.; Markovic, N. M., Nat Mater 2007, 6, 241-247.

8. Jasinski, R., Nature 1964, 201, 1212-1213.

9. Bashyam, R.; Zelenay, P., Nature 2006, 443, 63-66.

10. Chen, Z.; Higgins, D.; Yu, A.; Zhang, L.; Zhang, J., Energy Environ. Sci. 2011, 4, 3167-3192.

11. Jaouen, F.; Proietti, E.; Lefevre, M.; Chenitz, R.; Dodelet, J.-P.; Wu, G.; Chung, H. T.; Johnston, C. M.; Zelenay, P., Energy Environ. Sci. 2011, 4, 114-130.

12. Liu, G.; Li, X.; Lee, J.-W.; Popov, B. N., Catal. Sci. Technol. 2011, 1, 207217.

14. Wang, H.; Maiyalagan, T.; Wang, X., ACS Catal. 2012, 2, 781-794.

15. Bezerra, C. W. B.; Zhang, L.; Lee, K.; Liu, H.; Marques, A. L. B.; Marques, E. P.; Wang, H.; Zhang, J., Electrochim. Acta 2008, 53, 4937-4951.

16. Othman, R.; Dicks, A. L.; Zhu, Z., Int. J. Hydrog. Energy 2012, 37, 357-372. 49, 3972-3982. 447. 
20. Wu, G.; Johnston, C. M.; Mack, N. H.; Artyushkova, K.; Ferrandon, M.; Nelson, M.; Lezama-Pacheco, J. S.; Conradson, S. D.; More, K. L.; Myers, D. J.; Zelenay, P., J. Mater. Chem. 2011, 21, 11392-11405.

21. Charreteur, F.; Jaouen, F.; Ruggeri, S.; Dodelet, J.-P., Electrochim. Acta 2008, 53, 2925-2938.

22. Médard, C.; Lefèvre, M.; Dodelet, J. P.; Jaouen, F.; Lindbergh, G., Electrochim. Acta 2006, 51, 3202-3213.

23. Jaouen, F.; Lefèvre, M.; Dodelet, J.-P.; Cai, M., J. Phys. Chem. B 2006, 110, 5553-5558.

24. Bonakdarpour, A.; Lefevre, M.; Yang, R. Z.; Jaouen, F.; Dahn, T.; Dodelet, J. P.; Dahn, J. R., Electrochem. Solid State Lett. 2008, 11, B105-B108.

25. Jaouen, F.; Herranz, J.; Lefèvre, M.; Dodelet, J.-P.; Kramm, U. I.; Herrmann, I.; Bogdanoff, P.; Maruyama, J.; Nagaoka, T.; Garsuch, A.; Dahn, J. R.; Olson, T.; Pylypenko, S.; Atanassov, P.; Ustinov, E. A., ACS Appl. Mater. Interfaces 2009, 1, 1623-1639.

26. Proietti, E.; Jaouen, F.; Lefèvre, M.; Larouche, N.; Tian, J.; Herranz, J.; Dodelet, J.-P., Nat Commun 2011, 2, 416.

27. Kramm, U. I.; Herranz, J.; Larouche, N.; Arruda, T. M.; Lefevre, M.; Jaouen, F.; Bogdanoff, P.; Fiechter, S.; Abs-Wurmbach, I.; Mukerjee, S.; Dodelet, J.P., Phys. Chem. Chem. Phys. 2012, 14, 11673-11688.

28. Larouche, N.; Chenitz, R.; Lefèvre, M.; Proietti, E.; Dodelet, J.-P., Electrochim. Acta 2014, 115, 170-182.

29. Yang, L.; Larouche, N.; Chenitz, R.; Zhang, G.; Lefèvre, M.; Dodelet, J.-P., Electrochim. Acta 2015, 159, 184-197.

30. Zhao, Y.; Watanabe, K.; Hashimoto, K., J. Am. Chem. Soc. 2012, 134, 19528-19531.

31. Ziegelbauer, J. M.; Olson, T. S.; Pylypenko, S.; Alamgir, F.; Jaye, C.; Atanassov, P.; Mukerjee, S., J. Phys. Chem. C 2008, 112, 8839-8849.

32. Herranz, J.; Jaouen, F.; Lefèvre, M.; Kramm, U. I.; Proietti, E.; Dodelet, J.P.; Bogdanoff, P.; Fiechter, S.; Abs-Wurmbach, I.; Bertrand, P.; Arruda, T. M.; Mukerjee, S., J. Phys. Chem. C 2011, 115, 16087-16097.

33. Ramaswamy, N.; Mukerjee, S., Adv. Phys. Chem. 2012, 2012, 491604491621.

34. Li, X.; Liu, G.; Popov, B. N., J. Power Sources 2010, 195, 6373-6378. 
35. Li, X.; Popov, B. N.; Kawahara, T.; Yanagi, H., J. Power Sources 2011, 196, 1717-1722.

36. Tylus, U.; Jia, Q.; Strickland, K.; Ramaswamy, N.; Serov, A.; Atanassov, P.; Mukerjee, S., J. Phys. Chem. C 2014, 118, 8999-9008.

37. Nallathambi, V.; Lee, J.-W.; Kumaraguru, S. P.; Wu, G.; Popov, B. N., J. Power Sources 2008, 183, 34-42.

38. Liu, G.; Li, X.; Ganesan, P.; Popov, B. N., Appl. Catal. B: Environ. 2009, 93, 156-165.

39. Chen, J.; Cui, X.; Zheng, W., Catal. Commun. 2015, 60, 37-41.

40. Cheon, J. Y.; Kim, T.; Choi, Y.; Jeong, H. Y.; Kim, M. G.; Sa, Y. J.; Kim, J.; Lee, Z.; Yang, T. H.; Kwon, K.; Terasaki, O.; Park, G. G.; Adzic, R. R.; Joo, S. H., Sci Rep 2013, 3, 2715.

41. Jaouen, F.; Goellner, V.; Lefèvre, M.; Herranz, J.; Proietti, E.; Dodelet, J. P., Electrochim. Acta 2013, 87, 619-628.

42. Lu, A. H.; Schüth, F., Adv. Mater. 2006, 18, 1793-1805.

43. Joo, S. H.; Lee, H. I.; You, D. J.; Kwon, K.; Kim, J. H.; Choi, Y. S.; Kang, M.; Kim, J. M.; Pak, C.; Chang, H.; Seung, D., Carbon 2008, 46, 2034-2045.

44. Chang, H.; Joo, S. H.; Pak, C., J. Mater. Chem. 2007, 17, 3078-3088.

45. Zhang, J.; Wang, X.; Wu, C.; Wang, H.; Yi, B.; Zhang, H., React. Kinet. Catal. Lett. 2004, 83, 229-236.

46. Zeng, J.; Lee, J. Y.; Zhou, W., Appl. Catal. A: Gen. 2006, 308, 99-104.

47. Chen, J.; Jiang, C.; Yang, X.; Feng, L.; Gallogly, E. B.; Wang, R., Electrochem. Commun. 2011, 13, 314-316.

48. Ravela, B.; Newville, M., J. Synchrotron Rad. 2005, 12, 537-541.

49. Newville, M., J. Synchrotron Rad. 2001, 8, 322-324.

50. Rehr, J. J.; Albers, R. C., Phys. Rev. B 1990, 41, 8139-8149.

51. Rehr, J. J.; Albers, R. C., Reviews of Modern Physics 2000, 72, 621-654.

52. Garsany, Y.; Singer, I. L.; Swider-Lyons, K. E., Journal of Electroanalytical Chemistry 2011, 662, 396-406.

53. Kresse, G.; Furthmüller, J., Phys. Rev. B 1996, 54, 11169-11186.

54. Kresse, G.; Hafner, J., Phys. Rev. B 1993, 47, 558-561.

55. Blöchl, P. E., Phys. Rev. B 1994, 50, 17953-17979.

56. Perdew, J. P.; Burke, K.; Ernzerhof, M., Phys. Rev. Lett. 1996, 77, 38653868. 
57. Monkhorst, H. J.; Pack, J. D., Phys. Rev. B 1976, 13, 5188-5192.

440

58. Kuttiyiel, K. A.; Sasaki, K.; Choi, Y.; Su, D.; Liu, P.; Adzic, R. R., Energy Environ. Sci. 2012, 5, 5297-5304.

59. Jiang, Y.; Adams, J. B.; van Schilfgaarde, M., J. Chem. Phys. 2005, 123, 064701.

60. Kuttiyiel, K. A.; Choi, Y.; Hwang, S.-M.; Park, G.-G.; Yang, T.-H.; Su, D.; Sasaki, K.; Liu, P.; Adzic, R. R., Nano Energy 2015, 13, 442-449.

61. Wang, J. X.; Inada, H.; Wu, L.; Zhu, Y.; Choi, Y.; Liu, P.; Zhou, W.-P.; Adzic, R. R., J. Am. Chem. Soc. 2009, 131, 17298-17302. 


\section{Cover Image}




TOC Art



FeCo-OMPC(L)

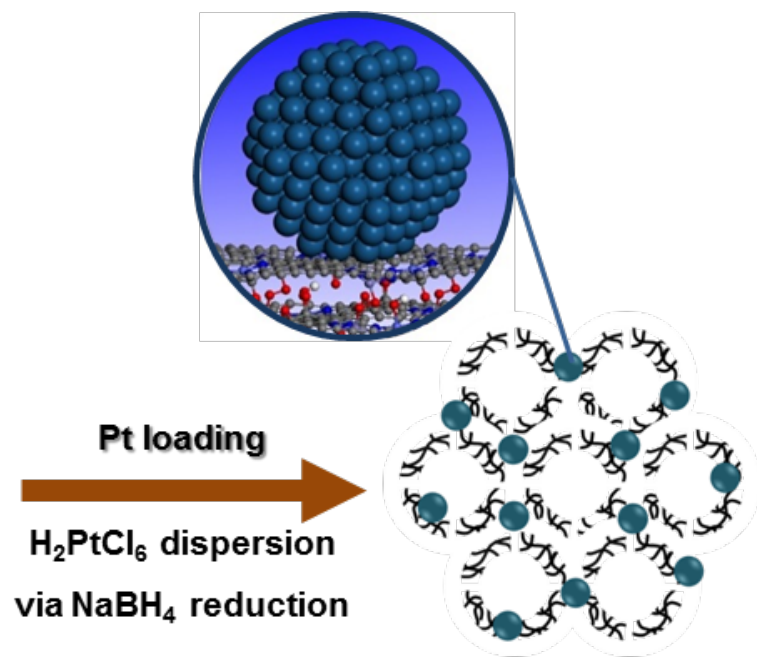

Pt/FeCo-OMPC(L) 


\section{Figure Legends}

Figure 1. (a) Synthetic process of Pt/FeCo-OMPC(L). TEM image of (b) OMS(L), (c) FeCo-OMPC(L), and (d) Pt/FeCo-OMPC(L), and EDS mapping images of (e) FeCoOMPC(L) and (f) $5 \mathrm{wt} \%$ Pt/FeCo-OMPC(L).

Figure 2. (a) Polarization curves for the ORR on prepared 5\% Pt/FeCo-OMPC(L) (4.49 $\left.\mu \mathrm{g}_{\mathrm{Pt}} / \mathrm{cm}^{2}\right), 5 \% \mathrm{Pt} / \mathrm{OMPC}(\mathrm{L})\left(6.22 \mu \mathrm{g}_{\mathrm{Pt}} / \mathrm{cm}^{2}\right)$, and 5\% Pt/C $\left(3.88 \mu \mathrm{g}_{\mathrm{Pt}} / \mathrm{cm}^{2}\right)$ catalysts in $\mathrm{O}_{2}$-saturated $0.1 \mathrm{M} \mathrm{HClO}_{4}$ with a sweep rate of $10 \mathrm{mV} / \mathrm{s}$ at $1600 \mathrm{rpm}$ (total catalyst loading is $84 \mu \mathrm{g}_{\text {catalyst }} / \mathrm{cm}^{2}$ ) and (b) comparison of mass and specific activities at $0.9 \mathrm{~V}$ vs. RHE.

Figure 3. (a) Polarization curves for the ORR on FeCo-OMPC(L), 5\% Pt/FeCoOMPC(L) (4.49 $\left.\mu \mathrm{g}_{\mathrm{Pt}} / \mathrm{cm}^{2}\right)$, and $\quad 10 \% \quad \mathrm{Pt} / \mathrm{FeCo-OMPC}(\mathrm{L}) \quad\left(6.63 \mu \mathrm{g}_{\mathrm{Pt}} / \mathrm{cm}^{2}\right)$ electrocatalysts in $\mathrm{O}_{2}$-saturated $0.1 \mathrm{M} \mathrm{HClO}_{4}$ with a sweep rate of $10 \mathrm{mV} / \mathrm{s}$ at 1600 $\mathrm{rpm}$ (total catalyst loading is $84 \mu \mathrm{g}_{\text {catalyst }} / \mathrm{cm}^{2}$ ) and (b) comparison of mass activities at $0.9 \mathrm{~V}$ vs. RHE for 3\%, 5\%, and 10\% Pt/FeCo-OMPC(L) and commercial $40 \mathrm{wt} \%$ $\mathrm{Pt} / \mathrm{C}$.

Figure 4. Trends of surface area and total pore volume of Pt/FeCo-OMPC(L) obtained from $\mathrm{N}_{2}$ adsorption/desorption isotherms.

Figure 5. XPS spectrums of N1s, $\mathrm{Pt}_{4 / 2}$ for FeCo-OMPC(L), 5\% Pt/FeCo-OMPC(L), and 10\% Pt/FeCo-OMPC(L).

Figure 6. Fourier-transform (FT) EXAFS of Fe k-edge, Co k-edge, and Pt Llll-edge from in-situ XAFS analysis under potentiostatic condition at $0.3 \mathrm{~V}$ vs. RHE for FeCoOMPC(L), 5\% Pt/FeCo-OMPC(L), and 10\% Pt/FeCo-OMPC(L) catalyst.

Figure 7. Schematic illustration of FeCo-OMPC(L) surfaces (a) before and (b) after the replacement of Co to Pt and (c) after the Pt nanocluster formation. For clarity, only the topmost layer is shown, and (d) DFT results of specific activities versus 
calculated binding energy of atomic oxygen on the Fe center without and with the Pt nanocatalyst. 
Figure 1



(b)



(e)



(c)



(d)






Figure 2



(b)




Figure 3

Figure 4




Figure 5

Figure 6

(a)



(b)



(c)




Figure 7

(a)

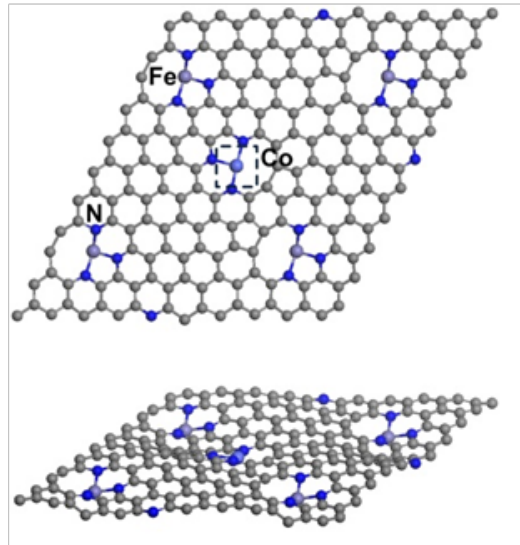

(c)



(b)



(d)




Table 1. Composition of prepared FeCo-OMPC(L), 5\% Pt/FeCo-OMPC(L), and 10\% Pt/FeCo-OMPC(L) catalysts

\begin{tabular}{c|ccc}
\hline & $\begin{array}{c}\text { FeCo-OMPC(L) } \\
\text { (wt.\% / at.\%) }\end{array}$ & $\begin{array}{c}\text { 5\% Pt/FeCo-OMPC(L) } \\
\text { (wt.\% / at.\%) }\end{array}$ & $\begin{array}{c}\text { 10\% Pt/FeCo-OMPC(L) } \\
\text { (wt.\% / at.\%) }\end{array}$ \\
\hline $\mathbf{P t}$ & - & $5.31 / 0.36$ & $7.85 / 0.54$ \\
$\mathbf{F e}$ & $1.63 / 0.36$ & $1.63 / 0.38$ & $1.49 / 0.36$ \\
$\mathbf{C o}$ & $1.47 / 0.31$ & $1.33 / 0.30$ & $1.10 / 0.25$ \\
$\mathbf{N}$ & $6.80 / 6.04$ & $5.90 / 5.51$ & $5.10 / 4.87$ \\
$\mathbf{C}$ & $90.10 / 93.29$ & $85.83 / 93.46$ & $84.46 / 93.99$ \\
\hline
\end{tabular}


Table 2. The structural parameters obtained from FT EXAFS curve-fitting process for the Fe, Co K-edge $\mathrm{k}^{2}$-weighted in-situ EXAFS spectra of the FeCo-OMPC(L), 5\% Pt/FeCo-OMPC(L), and 10\% Pt/FeCo-OMPC(L) catalysts

\begin{tabular}{|c|c|c|c|c|}
\hline Edge & Path & Catalysts & $R(\AA)^{a}$ & $N^{b}$ \\
\hline \multirow{3}{*}{ Co K-edge } & \multirow{3}{*}{ Co-N } & FeCo-OMPC(L) & 1.76 & 2.4 \\
\hline & & 5\% Pt/FeCo-OMPC(L) & 1.72 & 1.6 \\
\hline & & 10\% Pt/FeCo-OMPC(L) & 1.72 & 1.7 \\
\hline \multirow{3}{*}{ Fe K-edge } & \multirow{3}{*}{ Fe-N } & FeCo-OMPC(L) & 1.74 & 4.2 \\
\hline & & 5\% Pt/FeCo-OMPC(L) & 1.92 & 3.7 \\
\hline & & 10\% Pt/FeCo-OMPC(L) & 1.99 & 3.2 \\
\hline \multirow{4}{*}{$\mathrm{Pt} \mathrm{L}_{3}$-edge } & \multirow{2}{*}{ Pt-N } & 5\% Pt/FeCo-OMPC(L) & 1.93 & 1.4 \\
\hline & & 10\% Pt/FeCo-OMPC(L) & 2.01 & 0.6 \\
\hline & \multirow{2}{*}{ Pt-Pt } & 5\% Pt/FeCo-OMPC(L) & 2.85 & 7.6 \\
\hline & & $10 \% \mathrm{Pt} / \mathrm{FeCo}-\mathrm{OMPC}(\mathrm{L})$ & 2.86 & 7.4 \\
\hline
\end{tabular}

(a: bond distance, b: coordination number) 


\section{Supporting Information (SI)}

Figure S1. Polarization curves for the ORR on FeCo-OMPC(L), OMPC(L), and Vulcan XC-72 (VC) in $\mathrm{O}_{2}$-saturated $0.1 \mathrm{M} \mathrm{HClO}_{4}$ with a sweep rate of $10 \mathrm{mV} / \mathrm{s}$ at $1600 \mathrm{rpm}$.

Figure S2. Normalized (a) Fe k-edge and (b) Co k-edge XANES spectra for FeCoOMPC(L), 5\% Pt/FeCo-OMPC(L), and 10\% Pt/FeCo-OMPC(L) catalysts.

Figure S3. (a) The surface model with the (0001) surface plane consisting of 152 atoms (120 C, 12 N, 14 O, 2 Fe, 2 Co, and 2 H). The Co metal on the topmost layer was replaced by Pt to explain the Pt nanocluster growth. and (b) schematic side view of the FeCo-OMPC(L) surface without the M defect.

Figure S4. The bulk structure of FeCo-OMPC(L) consisting of 152 atoms (120 C, 12 N, 14 O, 2 Fe, 2 Co, and 2 H).

Figure S5. Schematic of the growth of Pt nanocluster on FeCo-OMPC(L). M and V are a metal center and its vacancy, respectively.

Figure S6. Schematic illustration of the growth of Pt nanocluster on FeCo-OMPC(L) with (a) $\mathrm{Pt}_{1}$, (b) $\mathrm{Pt}_{6}$, and (c) $\mathrm{Pt}_{19}$.

Figure S7. Specially designed in situ electrochemical cell for X-ray absorption spectroscopy (XAS) analysis. 
Figure S1




Figure S2

(a)



(b)




Figure S3

(a)



(b)




Figure S4

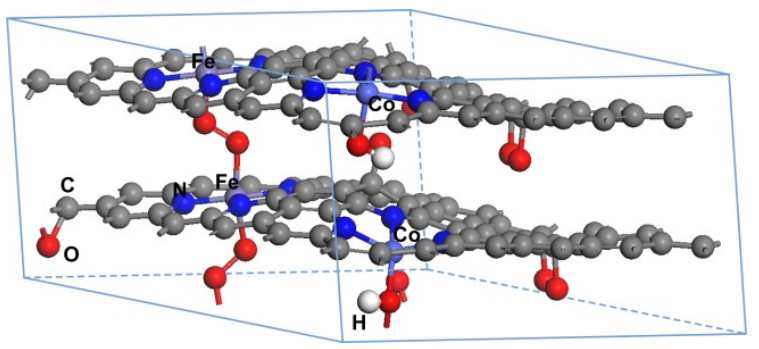


Figure S5

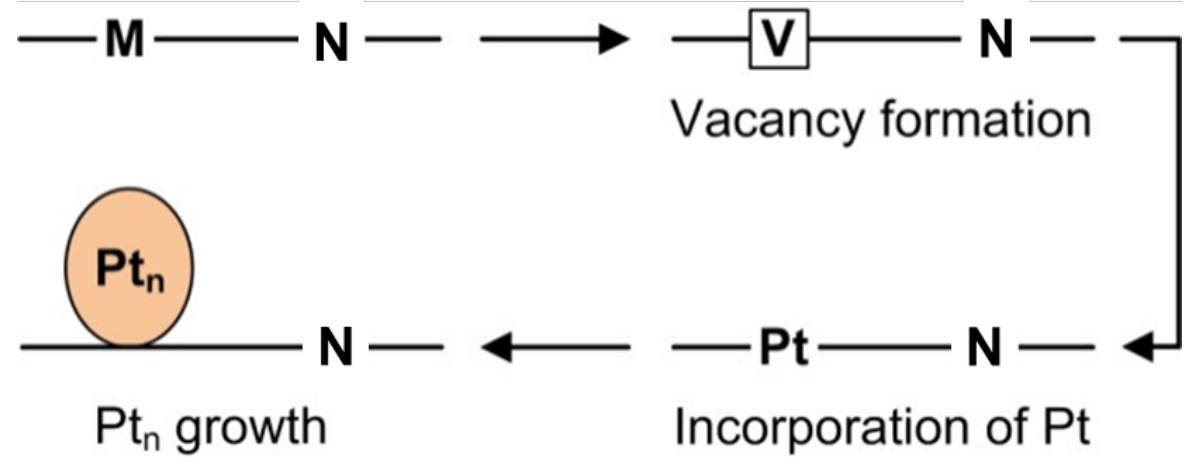


Figure S6

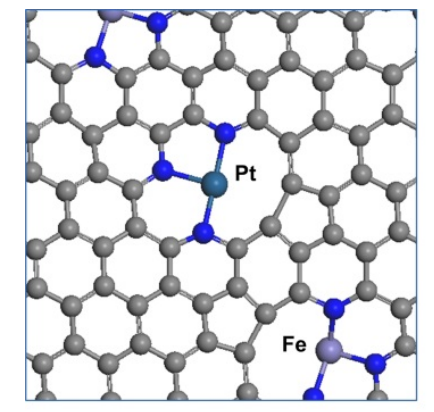

(a)

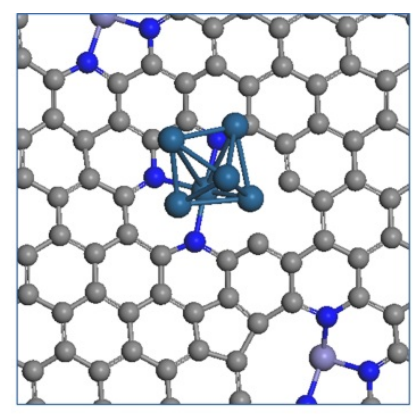

(b)

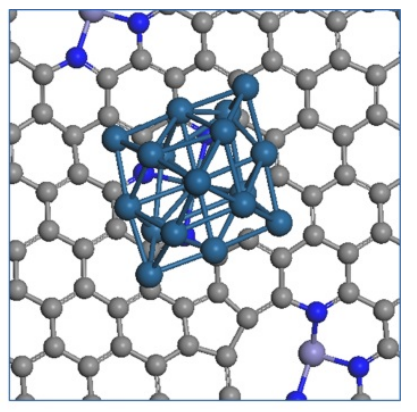

(c) 
Figure S7




Table S1. Comparison of Pt particle size $\left(\mathrm{d}_{\mathrm{Pt}}\right)$ calculated from XRD, and half-wave potentials $\left(\mathrm{E}_{1 / 2}\right)$, kinetic currents $\left(\mathrm{i}_{\mathrm{k}}\right)$, specific activity $\left(\mathrm{i}_{\mathrm{s}}\right)$, and mass activity $\left(\mathrm{i}_{\mathrm{m}}\right)$ at 0.9 $\mathrm{V}$ vs. RHE, and ECSA measured by CO stripping of the prepared catalysts

\begin{tabular}{|c|c|c|c|c|c|c|}
\hline Catalysts & $\begin{array}{c}d_{P t} \\
(n m)\end{array}$ & $\begin{array}{l}E_{1 / 2} \\
(m V)\end{array}$ & $\begin{array}{c}i_{k} \\
\left(m A / \mathrm{cm}^{2}\right)\end{array}$ & $\begin{array}{c}i_{s} \\
\left(\mu A / c^{2}\right)\end{array}$ & $\begin{array}{c}i_{m} \\
\left(A / m g_{P d}\right)\end{array}$ & $\begin{array}{l}E C S A \\
\left(m^{2} / g_{P}\right)\end{array}$ \\
\hline FeCo-OMPC(L) & - & 723 & 0.15 & - & - & - \\
\hline 5\% Pt/FeCo-OMPC(L) & 2.10 & 858 & 1.33 & 350 & 0.30 & 84.81 \\
\hline 10\% Pt/FeCo-OMPC(L) & 2.28 & 866 & 1.65 & 258 & 0.25 & 96.45 \\
\hline 5\% Pt/OMPC(L) & 3.50 & 845 & 0.94 & 157 & 0.15 & 96.58 \\
\hline $5 \% \mathrm{Pt} / \mathrm{C}$ & 6.80 & 755 & 0.16 & 50 & 0.04 & 80.50 \\
\hline
\end{tabular}


Table S2. The vacancy formation energies for the metal centers and incorporation energies of $\mathrm{Pt}$

\begin{tabular}{c|cc}
\hline & $E_{M-\text { vac }}(\text { eV/atom })^{a}$ & $E_{P t \text {-incorp }}(\mathrm{eV} / \text { atom })^{b}$ \\
\hline Co & 6.09 & -5.03 \\
Co, Fe & 6.34 & -4.73 \\
Fe & 6.74 & -4.80 \\
\hline
\end{tabular}

a. Vacancy formation energy of $\mathrm{M}$.

b. Incorporation energy of Pt atom into a metal vacancy. 
Table S3. Coordination number and geometrical parameters calculated using $\mathrm{DFT}^{\mathrm{a}}$

\begin{tabular}{c|ccccc}
\hline Surface & $N$ & $r_{(C o-N)}(\AA)$ & $r_{(F e-N)}(\AA)$ & $r_{(P t-N)}(\AA)$ & $r_{(P t-P t)}(\AA)$ \\
\hline Bulk OMPC(L) & 3 & 1.83 & 1.84 & - & - \\
$\mathrm{Pt}_{1} / \mathrm{FeCo}-\mathrm{OMPC}(\mathrm{L})_{\text {def }}$ & 3 & 1.83 & 1.87 & 1.93 & - \\
$\mathrm{Pt}_{6} / \mathrm{FeCo-OMPC}(\mathrm{L})_{\text {def }}$ & 3 & 1.83 & 1.87 & 1.95 & 2.58 \\
$\mathrm{Pt}_{19} / \mathrm{FeCo-OMPC}(\mathrm{L})_{\text {def }}$ & 3 & 1.83 & 1.87 & 1.97 & 2.64 \\
\hline
\end{tabular}

a. FeCo-OMPC $(\mathrm{L})_{\text {def }}$ is the surface with a Co vacancy on the topmost surface. 